\title{
Mobile health application to support CPAP therapy in obstructive sleep apnoea: design, feasibility and perspectives
}

\author{
Monique Suarez-Giron ${ }^{1,9}$, Onintza Garmendia ${ }^{1,2,9}$, Vera Lugo ${ }^{1}$, \\ Concepción Ruiz ${ }^{1}$, Neus Salord ${ }^{3,4}$, Xavier Alsina ${ }^{5}$, Ramón Farré2,6,7, \\ Josep M. Montserrat ${ }^{1,2,7,8}$ and Marta Torres ${ }^{1,2}$
}

\section{ABSTRACT}

Background: Current continuous positive airway pressure (CPAP) devices can be monitored remotely; however, in-person visits are kept for clinical follow-up in order to promote CPAP use and resolve potential side-effects. Mobile health is a promising way to provide remote and easy clinical control for CPAP follow-up and support. We aimed to evaluate the feasibility and acceptance by obstructive sleep apnoea (OSA) patients and healthcare professionals of a newly designed mobile app (Appnea-Q) to promote clinical control through a self-monitoring tool for patients with CPAP supervised by sleep professionals.

Methods: Appnea-Q incorporates a simple follow-up questionnaire with automated responses, together with frequent problems and lifestyle recommendations sections. Feasibility, acceptance and usefulness were assessed. First, an internal validation was performed during outpatient CPAP follow-up visits with sleep professionals from various sleep units. Second, an external validation was performed in a subgroup of 15 patients at home.

Results: Most patients $(n=75)$ considered the app useful and were willing to use it and recommend it (72-88\%). Up to $64.87 \%$ agreed on its capacity to reduce hospital visits. Appnea-Q was rated as acceptable $(79.37 \pm 19.29)$ by the system usability score. Sleep professionals $(n=30)$ concurred on its usefulness for OSA patient follow-up, particularly during the first month of CPAP therapy. The external validation showed its feasibility among 11 out of 15 patients and their data were received accordingly on the professionals' web platform.

Conclusions: According to our validation process, and the viewpoints of the patients and professionals, our new mobile app is a feasible and well-received tool for personal OSA management. Future clinical trials should substantiate its performance and cost-effectiveness in the clinical arena.

@ERSpublications

The implementation of mobile apps represents an attainable and fairly simple approach to support CPAP therapy initiation and follow-up management for sleep apnoea patients and healthcare professionals that can also be well received in the clinical field http://bit.ly/2rp4M1X

Cite this article as: Suarez-Giron M, Garmendia O, Lugo V, et al. Mobile health application to support CPAP therapy in obstructive sleep apnoea: design, feasibility and perspectives. ERJ Open Res 2020; 6: 00220-2019 [https://doi.org/10.1183/23120541.00220-2019].

This article has supplementary material available from openres.ersjournals.com

Received: 30 Aug 2019 | Accepted after revision: 4 Dec 2019

Copyright $\odot$ ERS 2020. This article is open access and distributed under the terms of the Creative Commons Attribution Non-Commercial Licence 4.0. 


\section{Introduction}

Obstructive sleep apnoea (OSA) is a highly prevalent chronic disease with serious morbidity associations and hence poses a major burden on the health system [1]. Continuous positive airway pressure (CPAP), the treatment of choice for OSA, is very effective but unfortunately suboptimal patient adherence to CPAP is common, as up to half of OSA patients either reject or discontinue CPAP within the first weeks. Accordingly, CPAP compliance represents a major challenge for sleep specialists $[2,3]$. Hence, strategic changes towards more cost-effective methods for managing the treatment of OSA with CPAP are urgently needed. As recently suggested, the use of telemedicine has significant potential for the management of patients with OSA [4-6].

Several clinical trials have demonstrated the feasibility of telemedicine-based OSA management compared with a more traditional, in-person care model, suggesting noninferiority in terms of adherence to CPAP treatment [7], CPAP compliance [8] as well as functional outcomes such as satisfaction [9] and cost-effectiveness $[10,11]$. In our previous experience using telemedicine for OSA management, we found positive feedback with videoconference and mobile health (mHealth) interventions [12, 13]. We also conducted a telemedicine-based strategy for CPAP follow-up which was as effective as standard hospital-based care in terms of CPAP compliance and symptoms improvement, as well as lower costs [11].

Nevertheless, technological innovations in healthcare can be potentially disadvantageous if they are not properly applied or understood, a phenomenon we have previously described as the "technological labyrinth syndrome" [14], basically referring to a process which is too complex for patients to follow or excessively time consuming for healthcare personal to follow-up. Therefore, it seems imperative that any new technology applied for health purposes is fundamentally simple, easy to use, reliable and transparent, and able to control or contact with the patient. mHealth is a modality of telemedicine that delivers a promising approach for patients through the use of portable devices such as smartphones and tablets for medical purposes, including treatment follow-up and support of general health. By means of mobile apps, patients' input can be gathered from interactive questionnaires and processed by specific algorithms to generate customised treatment recommendations [15]. From previous work we found that simple mHealth interventions are well received by patients and seem promising to improve CPAP compliance among patients with OSA [13]. mHealth is currently on the rise, following the wide spread of the internet and mobile devices. Given the increase in the development of mHealth apps, the potential reach and impact over health promotion have never been greater. Additionally, the potential impact on healthcare cost reductions should be considered as a positive outcome of using mHealth [4]. Nevertheless, further extensive studies are needed, and the opinions of both healthcare professionals and patients are essential before moving forward in the clinical implementation of telemedicine strategies [16].

Therefore, we have now designed an innovative mobile app called Appnea-Q (Appnea-Questions) with new functionalities that deliver a self-monitoring tool for OSA patients treated with CPAP aimed to promote easy and remote clinical control. So far, numerous providers have developed tools for CPAP monitoring mainly focused on the technological aspects of treatment, neglecting the clinical aspects of OSA patients treated with CPAP, which is of paramount importance during follow-up to ensure appropriate adaptation and compliance to the treatment. Previous work from the Spanish Sleep Network suggests a better CPAP compliance $\left(>5.6 \mathrm{~h} \cdot\right.$ night $\left.^{-1}\right)$ might help reduce the deleterious cardiovascular effects of OSA [17]. Here, we describe the features of the app, and evaluate its feasibility and acceptance by current OSA patients treated with CPAP and by professionals specialised in sleep medicine.

\section{Materials and methods}

Mobile application: patient interface

Appnea-Q requires the patient to login with a personal username and password for data recognition and privacy protection. Compared with our previous work in mHealth [13], in this app we have incorporated new

Affiliations: 'Laboratori del Son, Servei de Pneumologia, Hospital Clínic, Barcelona, Spain. ${ }^{2}$ Centro de Investigación Biomédica en Red de Enfermedades Respiratorias (CIBERES), Madrid, Spain. ${ }^{3}$ Sleep Unit, Dept of Respiratory Medicine, Hospital Universitari de Bellvitge, Hospitalet de Llobregat, Barcelona, Spain. ${ }^{4}$ Bellvitge Biomedical Research Institute (IDIBELL), Respiratory Medicine Section, Hospitalet de Llobregat, Barcelona, Spain. ${ }^{5}$ Dept of Pneumology and Respiratory Allergy, Hospital Clínic, Barcelona, Spain. ${ }^{6}$ Unitat de Biofísica i Bioenginyeria, Facultat de Medicina i Ciències de la Salut, Universitat de Barcelona, Barcelona, Spain. ${ }^{7}$ Institut d'Investigacions Biomèdiques August Pi i Sunyer, Barcelona, Spain. ${ }^{8}$ Departament de Medicina, Facultat de Medicina i Ciències de la Salut, Universitat de Barcelona, Barcelona, Spain. ${ }^{9}$ These authors contributed equally to this work.

Correspondence: Josep M. Montserrat, Servei de Pneumologia, Hospital Clínic, Villarroel 170, 08036 Barcelona, Spain. E-mail: jcanaldclinic.cat 
features and more information focused on the patient's needs to deliver a self-monitoring tool divided into three main sections: 1) follow-up questionnaire, 2) frequent problems and 3) recommendations (figure 1).

The follow-up questionnaire section of Appnea-Q consists of 10 questions in total, nine of them single-choice questions (Yes/No) divided into the following aspects of treatment: CPAP use and effectiveness (Questions 1-3), common side-effects (Questions 4-6), exercise and diet (Questions 7-9), and a final question to fill in the patient's current weight (Question 10). In addition, Appnea-Q provides automated feedback according to the patient's answers. Nonsatisfactory answers receive useful advice, while positive answers receive an encouraging message (figure 2 and supplementary table S1). Patients can receive periodic reminders set by the physician to complete the questionnaire, as often as needed.

The frequent problems section of Appnea-Q summarises the most common side-effects and issues faced by CPAP patients during the course of the treatment (e.g. dry mouth, air leaks and nasal congestion) (supplementary figure S1). The main goal is to provide useful solutions, with the patient being allowed to consult at any time a problem arises, gaining valuable time and supporting their CPAP therapy. In case the problem is not resolved, a voicemail contact is provided so patients can deliver consultations that will be attended by the sleep unit nurses within $24-48 \mathrm{~h}$

In the recommendations section, Appnea-Q users can find general information concerning CPAP use, sleep hygiene rules and diet information. This information is aimed at promoting an integral and more effective treatment approach to OSA. All components in the app have been specifically designed by our team of specialised nurses and physicians.

The Spanish version of Appnea-Q is available for free download on Android platforms via Google Play and for iOS, respectively:

https://play.google.com/store/apps/details?id=net.bdevelopments.appneaQuestions and https://itunes.apple. com/us/app/appneaquestions/id1404229790?platform=iphone\&preserveScrollPosition=true\&platform= iphone\#platform/iphone\&platform=iphone

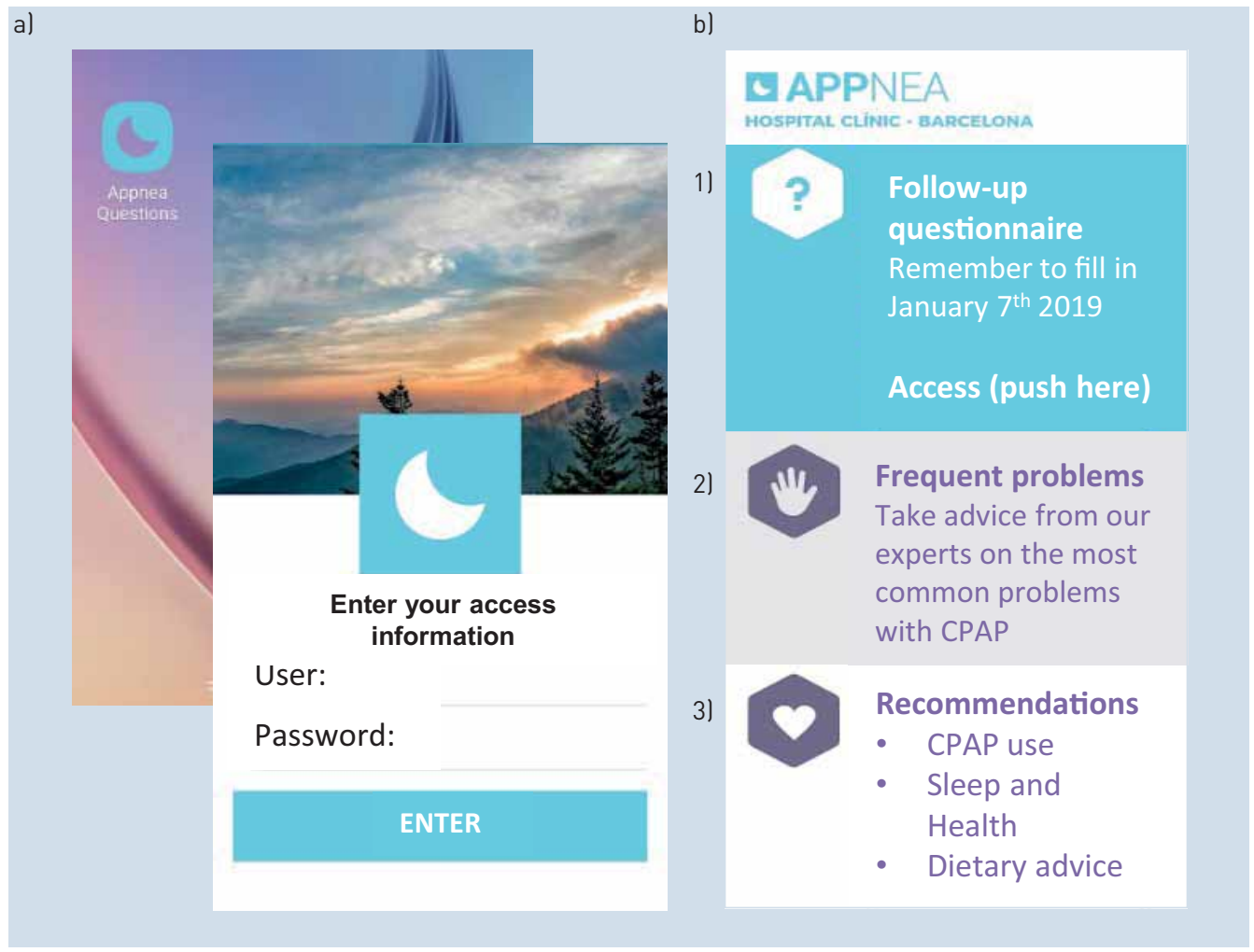

FIGURE 1 a) Appnea-Q smartphone access and patient password-protected login screen and b) main screen, divided into three sections: 1) follow-up questionnaire, 2) frequent problems and 3) recommendations. CPAP: continuous positive airway pressure. The original text in Spanish has been translated into English. 


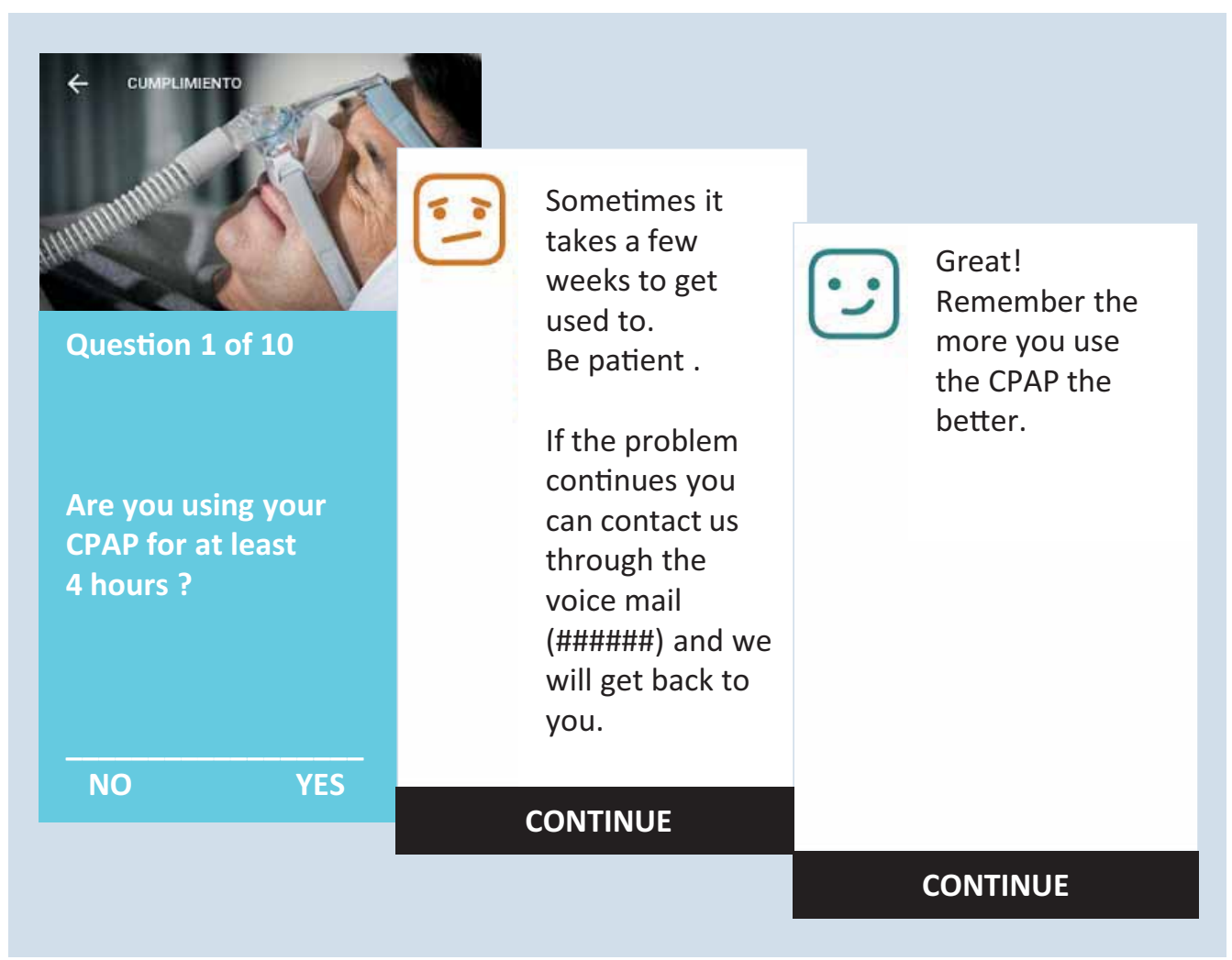

FIGURE 2 Example follow-up question: automated answers give advice to patients. CPAP: continuous positive airway pressure. The original text in Spanish has been translated into English.

\section{Web platform: sleep care professionals}

We designed a password-protected web platform where sleep professionals can login from their sleep unit. The app content can be accessed with a username and password registered by hospital professionals only. Connections to the server are made under a secure SSL connection protocol, which means that the information transmitted is encrypted. Professionals can create as many personal users as they need, for those patients they decide to monitor through the app. Each patient is categorised by the sleep unit that is attending him/her. The sleep professional can review the information gathered from all their patients' questionnaires on this web platform, and a filter is applied so those with nonsatisfactory responses will appear at the top of the patients list and will be marked in red, allowing the hospital professional to easily identify patients in need of early assistance (supplementary figure S2). Once the questionnaire has been reviewed, the patient's name is returned to the chronological order it was created.

\section{Feasibility and acceptance of Appnea-Q}

This trial was conducted in the Hospital Clínic (Barcelona, Spain), where it was approved by the relevant Ethics Committee. To evaluate the feasibility and acceptance of Appnea-Q, 75 consecutive patients on CPAP follow-up consultations were asked to use the app during the visit, answering the questionnaire and exploring the frequent problems and recommendations sections. Patients were recruited from two tertiary hospitals. All patients received a personal username and password to sign up, and their answers were tracked by the professionals' web platform. After testing the app, all patients completed a System Usability Scale (SUS), a Likert-type scale used to assess the user's subjective view of the app usability [18], followed by a questionnaire on the level of satisfaction with the app content. An external validation was also performed in 15 out of the 75 patients recruited, installing the app on their own smartphone and asking them to use it during 3 consecutive days, tracking patients' answers to the follow-up questionnaires and submitting a satisfaction survey afterwards.

Additionally, sleep healthcare professionals were recruited from 15 different sleep units from all over Spain. All specialised sleep physicians and nurses/technicians were asked to explore Appnea-Q and answer a short survey on its usability to follow-up OSA patients. A descriptive analysis was conducted. Quantitative variables were assessed by calculating the arithmetic mean and standard deviation. For qualitative variables, percentages were calculated. 


\section{Results}

Internal validation

Patients

75 patients with OSA under CPAP treatment were enrolled. Most of the patients were male (84\%), with middle to superior studies (81.33\%) and reported frequent use of smartphones $(85.33 \%)$; their baseline characteristics are summarised in table 1. Only five patients had trouble completing the questionnaire. Remarkably, they were all aged $>75$ years old. Only three patients could not finalise the questionnaire because of a technical failure when introducing their weight. SUS score was rated on the subjective scale as good; according to the assessments of the usability scale it was rated as acceptable with a score of $79.37 \pm 19.29$ and a 50th percentile of 82.5. Moreover, on a linear scale from 1 to 10 , the utility of Appnea-Q was rated at 7.70.

When evaluating Appnea-Q content, 75 patients answered the satisfaction survey; most considered that the different app sections were useful. Specifically, the frequent problems section was considered the most useful (88\%), followed by the diet, healthy sleep and CPAP use section $(82.67 \%)$, and automated questionnaire answers (74\%). Additionally, most of the patients would recommend the app to other users (86.49\%) and considered it useful to control their OSA condition (73.33\%). Moreover, in relation to the capacity of Appnea-Q to reduce hospital visits, $64.87 \%$ agreed, while the rest were not sure $(22.97 \%)$ or did not consider it possible (12.16\%). The patients' survey is summarised in table 2.

\section{Professionals}

30 healthcare professionals specialised in sleep respiratory medicine (physicians $\mathrm{n}=17$ and nurses/ technicians $\mathrm{n}=13$ ) from different sleep units all over Spain explored Appnea-Q and then answered a short survey (table 3). They all concurred that Appnea-Q would be useful to provide OSA patient control and follow-up, particularly during the first month of CPAP therapy initiation, and routine follow-up as a second option. They also agreed that the app could reduce the number of hospital visits. Neither physicians nor nurses/technicians showed major concerns about the risk of workplace reduction as a consequence of incorporating the app in the clinical routine (results are summarised in table 3 ).

\section{External validation: patients}

An external validation was performed in a subgroup of 15 patients (out of the original 75 patients) that used the app for 3 consecutive days in their home. All were male, mean age $61.2 \pm 7.68$ years, apnoea-hypopnoea index $45 \pm 21.37$ events $\cdot \mathrm{h}^{-1}$, CPAP use $5.67 \pm 3.20 \mathrm{~h} \cdot$ night $^{-1}$ and $86.66 \%$ with middle to superior studies, and all 15 reported frequent use of smartphones. Their mean SUS score was $85.17 \pm 14.25$; rated on the subjective scale as excellent and on the usability scale as acceptable. 11 out of 15 patients completed the follow-up questionnaire of the first day and second day, and nine out of 15 patients also completed the follow-up questionnaire of the third day. Data were received accordingly on the professionals' web platform. Additionally, the satisfaction survey for this group included a question regarding the periodicity over which they wished to answer the questionnaire during their CPAP therapy follow-up; the opinion was evenly divided between weekly, monthly or twice a week.

\section{Discussion}

Our findings suggest that the mobile app Appnea-Q is a feasible and well-received tool for OSA management according to patients and sleep professionals. On the one hand, the content and technological usability of the app was positively valued by OSA patients. On the other hand, healthcare

\section{TABLE 1 Baseline patient characteristics}

Patients

Male

Age years

BMI $\mathrm{kg} \cdot \mathrm{m}^{-2}$

AHI events $\cdot h^{-1}$

CPAP compliance $h \cdot$ night $^{-1}$

Middle to superior studies

Frequent use of smartphones

Data are presented as $n, n(\%)$ or mean \pm SD. BMI: body mass index, AHI: apnoea-hypopnoea index; CPAP: continuous positive airway pressure. 


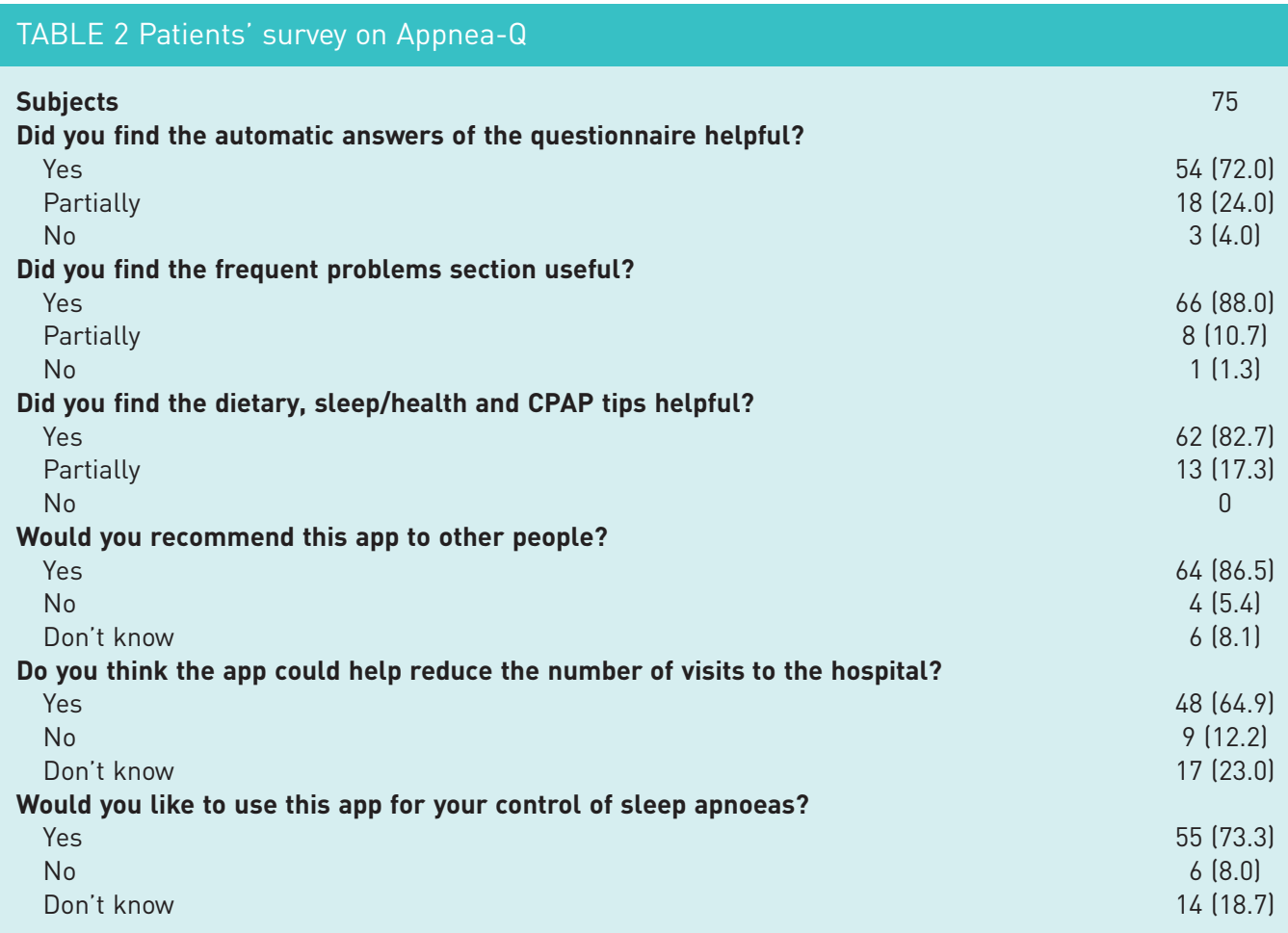

Data are presented as $n$ or $n(\%)$; percentages may not total $100 \%$ due to rounding. CPAP: continuous positive airway pressure.

TABLE 3 Sleep healthcare professionals' survey on Appnea-Q usefulness and impact on hospital visits and workplaces

\begin{tabular}{lcc} 
& $\begin{array}{c}\text { Sleep } \\
\text { physicians }\end{array}$ & $\begin{array}{c}\text { Sleep nurses/ } \\
\text { technicians }\end{array}$ \\
\hline $\begin{array}{l}\text { Subjects } \\
\text { Do you think that this app can be useful for control and }\end{array}$ & 17 \\
monitoring of patients with sleep apnoea? & & \\
Yes & $17(100)$ & $12(92.3)$ \\
No & 0 & $1(7.7)$ \\
Don't know & 0 & 0 \\
Do you think that using this app could reduce the number of & & \\
hospital visits? & $11(64.7)$ & $9(69.2)$ \\
Yes & 0 & $1(7.7)$ \\
No & $6(35.3)$ & $3(23.1)$ \\
Don't know & & $12(92.3)$ \\
In what situations do you think this app could be useful? & $15(88.2)$ & $3(23.1)$ \\
(Multiple choices) & $4(23.5)$ & $7(53.8)$ \\
First month of CPAP treatment & $8(47.1)$ & \\
Non-compliant patients & & $2(15.4)$ \\
Routine follow-up & & $6(46.1)$ \\
Do you think that this system can reduce jobs because of more & $2(11.8)$ & $5(38.5)$ \\
telematic contact but less hospital visits? & $10(58.8)$ & $5(29.4)$ \\
Yes & & \\
No & & \\
Don't know & & \\
&
\end{tabular}

Data are presented as $n$ or $n(\%)$; percentages may not total $100 \%$ due to rounding. CPAP: continuous positive airway pressure. 
professionals found the app could be useful to manage OSA patients and to reduce in-hospital visits, particularly during the first month of CPAP therapy.

Telemedicine and particularly mHealth are suitable strategies that could transform the healthcare approach by providing increased and anytime access to information, and empower patients to be informed, engaged and equipped to take part in shared decision making and effective self-management of chronic illnesses [19], shifting healthcare from a disease-centred to a patient-centred model [20]. The use of telemedicine to enhance CPAP follow-up is an area that has been most explored, but the optimal format, information and delivery mechanism are still unclear [21].

Our previous experiences in telemedicine and OSA management have involved a series of telemedicine strategies, a very enlightening process in the search for the most adequate strategy. Initially, patients were satisfied with videoconference interventions for CPAP follow-up consultation and CPAP training sessions [12]. Later, a telemedicine-based strategy for CPAP follow-up compared with in-hospital-based care revealed similar levels of CPAP compliance, and improved symptoms, side-effects, quality of life and degree of satisfaction. In addition, the telemedicine-based strategy had lower total costs due to savings on transport and less lost productivity [11]. Furthermore, we developed an early mHealth app which was shown to be feasible and satisfactory in OSA patients [13]. More recently, we evaluated patients' perspective on a new management approach for CPAP titration encompassing both telemedicine and mHealth compared with in-hospital-based care. Using a focus group methodology, most patients described the telemedicine approach as an innovative, accessible and time-saving way to control their treatment, which was also found to be feasible in patients with limited knowledge of the use of the internet and mobile apps [22]

Consequently, our findings confirmed that the telemedicine patient's opinion is of paramount importance when designing future telemedicine procedures, and that these telemedicine approaches can also be troublesome if they are not properly validated and applied to the correct population targets. In fact, they could even increase mortality in certain groups [23-25]. Therefore, ease of use, usefulness, effectiveness, low cost and reliability are paramount in telemedicine interventions [23]. To that end, telemedicine interventions must be enlightened by more research on their usability by both patients and healthcare professionals [26]. Even though telemedicine is in general well accepted by OSA patients [27], recent work by TURINo et al. [10] showed telemonitoring of CPAP signals alone did not improve CPAP treatment compliance and was associated with lower patient satisfaction. Moreover, from the professionals' side there are well-known factors preventing the widespread implementation of this strategy, including poor organisation in healthcare institutions, regulations and clinical practice guidelines, and staff training. In addition, the accessibility to these resources and the sense of self-efficacy are also factors related to the use of telemedicine. One of the main resistance factors to the use of telemedicine is the idea from health professionals of a potential increase in work overload. However, different studies have shown that both the implementation of clear protocols and previous staff training have positive effects [28, 29]. Overcoming these resistance factors could open the door to a more pro-active telemedicine implementation, facilitating the decentralisation of services [4].

Currently, some CPAP device manufacturers provide mHealth web platforms allowing physicians and patients to remotely receive informative data such as compliance, apnoea-hypopnoea index and mask leaks, and present data in different ways (e.g. Encore Anywhere (Philips) and AirView (ResMed)). Moreover, each company employs its own proprietary algorithm, complicating the interpretation of results by health professionals [30]. Despite this technological aspect, which is certainly useful, personal interactions between patients and healthcare professionals are also essential in telemedicine interventions, since patient customisation is required [31-33]. In this context, Appnea-Q has been developed for personal interactions between patients and professionals to be useful in a significant number of subjects regardless of their phenotype.

Recently, Hwang et al. [8] conducted a trial with 1455 OSA patients and found that combining two telemedicine (educational and telematics data) interventions increased CPAP usage to $\sim 4.8 \mathrm{~h} \cdot \mathrm{night}^{-1}$ compared with conventional treatment $\left(\sim 3.8 \mathrm{~h} \cdot\right.$ night $\left.^{-1}\right)$. However, such an ambitious approach does not distinguish the usefulness of the new intervention in specific patient groups. Hence, it seems wise to remain cautious and conduct targeted evaluations that help consolidate the role of telemedicine in routine CPAP follow-up [34]. Although supportive interventions are reported to increase usage in CPAP-naive patients, there is little evidence in people who have struggled with CPAP [35]. We must keep in mind that if telemedicine delivery to OSA patients' follow-up is efficacious it could have a potential impact on healthcare utilisation and a subsequent reduction in costs [36]. As these new telemedicine strategies are explored, it is of paramount importance that physicians see them as a manageable, reliable and safe asset to their practice. Meanwhile, patients' technological barriers and quality concerns need to be openly faced and overcome to provide a simple and easy to use system [30]. 
In conclusion, our mobile app can be a well-received tool in the strategy to monitor and improve clinical control of the OSA patient's chronic condition and their therapy with CPAP, to encourage healthy lifestyles, with active participation of both patients and professionals. However, full clinical validation of this telemedicine strategy dedicated to OSA management is mandatory before its proper application into general routine practice.

Acknowledgements: The authors wish to thank Gemma Guerrero and Cristina Cerrato (Hospital Clínic, Barcelona, Spain) and Hermes Carretero (Web Developer, Barcelona, Spain) for their assistance.

Support statement: This work was funded by the Carlos III Institute of Health, Ministry of Economy and Competitiveness (Spain) (PI17/01068). Funding information for this article has been deposited with the Crossref Funder Registry.

Conflict of interest: M. Suarez-Giron has nothing to disclose. O. Garmendia has nothing to disclose. V. Lugo has nothing to disclose. C. Ruiz has nothing to disclose. N. Salord has nothing to disclose. X. Alsina has nothing to disclose. R. Farre reports contracts between the University of Barcelona, ResMed and ANTADIR to evaluate CPAP devices at the bench. J.M. Montserrat reports an official government grant from the Ministry of Economy Industry and Competitiveness, and that Philips Respironics supported one of the researchers. M. Torres has nothing to disclose.

\section{References}

1 Young T, Palta M, Dempsey J, et al. Burden of sleep apnea: rationale, design, and major findings of the Wisconsin Sleep Cohort study. WMJ 2009; 108: 246-249.

2 Osman AM, Carter SG, Carberry JC, et al. Obstructive sleep apnea: current perspectives. Nat Sci Sleep 2018; 10: $21-34$.

3 Engleman HM, Wild MR. Improving CPAP use by patients with the sleep apnoea/hypopnoea syndrome. Sleep Med Rev 2003; 7: 81-99.

4 Isetta V, Ruiz M, Farré $\mathrm{R}$, et al. Supporting patients receiving CPAP treatment: the role of training and telemedicine. In: Barbé F, Pépin JL, eds. Obstructive Sleep Apnoea (ERS Monograph). Sheffield, European Respiratory Society, 2015; pp. 280-292.

5 Zia S, Fields BG. Sleep telemedicine: an emerging field's latest frontier. Chest 2016; 149: 1556-1565

6 Dorsey ER, Topol EJ. State of telehealth. N Engl J Med 2016; 375: 154-161.

7 Hoet F, Libert W, Sanida C, et al. Telemonitoring in continuous positive airway pressure-treated patients improves delay to first intervention and early compliance: a randomized trial. Sleep Med 2017; 39: 77-83.

8 Hwang D, Chang JW, Benjafield AV, et al. Effect of telemedicine education and telemonitoring on continuous positive airway pressure adherence. The Tele-OSA randomized trial. Am J Respir Crit Care Med 2018; 197: $117-126$.

9 Fields BG, Behari PP, McCloskey S, et al. Remote ambulatory management of veterans with obstructive sleep apnea. Sleep 2016; 39: 501-509.

10 Turino C, De Batlle J, Woehrle H, et al. Management of continuous positive airway pressure treatment compliance using telemonitoring in obstructive sleep apnoea. Eur Respir J 2017; 49: 1601128.

11 Isetta V, León C, Torres M, et al. Telemedicine-based approach for obstructive sleep apnea management: building evidence. J Med Internet Res 2014; 16: 1-12.

12 Isetta $\mathrm{V}$, Torres $\mathrm{M}$, González $\mathrm{K}$, et al. A new mHealth application to support treatment of sleep apnoea patients. J Telemed Telecare 2017; 23: 14-18.

13 Isetta V, Negrín MA, Monasterio C, et al. A Bayesian cost-effectiveness analysis of a telemedicine-based strategy for the management of sleep apnoea: a multicentre randomised controlled trial. Thorax 2015; 70: 1054-1061.

14 Suarez-Giron MC, Isetta V, Masa JF, et al. Sleep breathing disorders: have we reached the tipping point? ERJ Open Res 2018; 4: 00172-2017.

15 Cortez NG, Cohen IG, Kesselheim AS. FDA regulation of mobile health technologies. N Engl J Med 2014; 371: 372-379.

16 O'Connor S, Hanlon P, O'Donnell CA, et al. Understanding factors affecting patient and public engagement and recruitment to digital health interventions: a systematic review of qualitative studies. BMC Med Inform Decis Mak 2016; 16: 120.

17 Martínez-García MA, Capote F, Campos-Rodríguez F, et al. Effect of CPAP on blood pressure in patients with obstructive sleep apnea and resistant hypertension: the HIPARCO randomized clinical trial. JAMA 2013; 310: 2407-2015.

18 Brooke J. SUS: a retrospective. J Usability Studies 2013; 8: 29-40.

19 Macdonald GG, Townsend AF, Adam P, et al. eHealth technologies, multimorbidity, and the office visit: qualitative interview study on the perspectives of physicians and nurses. J Med Internet Res 2018; 20: e31.

20 Zulman DM, Jenchura EC, Cohen DM, et al. How can eHealth technology address challenges related to multimorbidity? Perspectives from patients with multiple chronic conditions. J Gen Intern Med 2015; 30: 1063-1070.

21 Hwang D. Monitoring progress and adherence with positive airway pressure therapy for obstructive sleep apnea: the roles of telemedicine and mobile health applications. Sleep Med Clin 2016; 11: 161-171.

22 Garmendia O, Suarez-Giron MC, Torres M, et al. Telemedicine in sleep apnea: a simple approach for nasal pressure (CPAP) treatment. Arch Bronconeumol 2018; 54: 491-492.

23 Kruse CS, Krowski N, Rodriguez B, et al. Telehealth and patient satisfaction: a systematic review and narrative analysis. BMJ Open 2017; 7: e016242.

24 Lee-Tobin PA, Ogeil RP, Savic M, et al. Rate my sleep: examining the information, function, and basis in empirical evidence within sleep applications for mobile devices. J Clin Sleep Med 2017; 13: 1349-1354.

25 Takahashi PY, Pecina JL, Upatising B, et al. A randomized controlled trial of telemonitoring in older adults with multiple health issues to prevent hospitalizations and emergency department visits. Arch Intern Med 2012; 172: 773-779. 
Klaassen B, Van Beijnum BJ, Hermens HJ. Usability in telemedicine systems - a literature survey. Int J Med Inform 2016; 93: 57-69.

27 Bros JS, Poulet C, Arnol N, et al. Acceptance of telemonitoring among patients with obstructive sleep apnea syndrome: how is the perceived interest by and for patients? Telemed J E Health 2018; 24: 351-359.

28 Carter M, Fletcher E, Sansom A, et al. Feasibility, acceptability and effectiveness of an online alternative to face-to-face consultation in general practice: a mixed-methods study of webGP in six Devon practices. BMJ Open 2018; 8: e018688.

29 Segrelles-Calvo G, Chiner E, Fernández-Fabrellas E. Acceptance of telemedicine among healthcare professionals Arch Bronconeumol 2015; 51: 611-612.

30 Verbraecken J. Telemedicine applications in sleep disordered breathing: thinking out of the box. Sleep Med Clin 2016; 11: 445-459.

31 Welch BM, Harvey J, O'Connell NS, et al. Patient preferences for direct-to-consumer telemedicine services: a nationwide survey. BMC Health Serv Res 2017; 17: 784

32 Runz-Jørgensen SM, Schiøtz ML, Christensen U. Perceived value of eHealth among people living with multimorbidity: a qualitative study. J Comorb 2017; 7: 96-111.

33 Cimperman M, Brenčič MM, Trkman P, et al. Older adults' perceptions of home telehealth services. Telemed J E Health 2013; 19: 786-790.

34 Farré R, Navajas D, Montserrat JM. Is telemedicine a key tool for improving continuous positive airway pressure adherence in patients with sleep apnea? Am J Respir Crit Care Med 2018; 197: 12-14.

35 Wozniak DR, Lasserson TJ, Smith I. Educational, supportive and behavioural interventions to improve usage of continuous positive airway pressure machines in adults with obstructive sleep apnoea. Cochrane Database Syst Rev 2014; 1: CD007736.

36 Villanueva JA, Suarez MC, Garmendia O, et al. The role of telemedicine and mobile health in the monitoring of sleep-breathing disorders: improving patient outcomes. Smart Homecare Technol Telehealth 2017; 4: 1-11. 